The authors of this study conclude that appropriate indications and timing of AED level determinations were not followed in $75 \%$ of patients in their tertiary care hospital. More careful adherence to appropriate monitoring indications would have resulted in cost reductions without significant risk of ineffective therapy or toxicity.

\title{
AGE FACTORS AND ANTIEPILEPTIC DRUG WITHDRAWAL
}

Age dependent factors concerning the withdrawal of antiepileptic drugs (AED) and seizure relapse rates, after a seizure-free period longer than 3 years, were evaluated in 304 patients with childhood epilepsies treated at the Toyama Medical and Pharmaceutical University, Toyama City, Japan. The incidence of AED withdrawal differed significantly between epileptic syndromes, being higher in idiopathic than in symptomatic epilepsies. Age at withdrawal peaked at preadolescence and early school age. Relapses occurred in $14 \%$, the rate differing between epileptic groups and occurring at a unique age in each epileptic syndrome. Relapse rates were $33 \%$ and $20 \%$ in symptomatic generalized and partial epilepsies, respectively, and 5 to $8 \%$ in benign infantile convulsions, and idiopathic partial epilepsies. Idiopathic generalized epilepsy syndromes had higher relapse rates: $25 \%$ in juvenile absence, $100 \%$ in juvenile myoclonic, and $27 \%$ in grand mal on awakening epilepsy. Relapses were more frequent in epilepsies of infantile or adolescent onset than in those of school age onset. Age of relapse peaked at ages 7 to 11 , mainly benign childhood epilepsy with centrotemporal spikes, and 17 to 19 years, mainly symptomatic partial, juvenile myoclonic, and grand mal.

EEG paroxysmal discharges did not necessarily predict a relapse, but changes in background activity with age showed correlations with rate of seizure recurrence. In patients without relapse, the background showed an increased maturation in mean frequency, with decrease in slow waves and increased alpha activity, during AED control before drug withdrawal. (Murakami M et al. Withdrawal of antiepileptic drug treatment in childhood epilepsy: factors related to age. L Neurol Neurosurg Psychiatry 1995;59:477481). (Respond: Dr Miyako Murakami, Department of Pediatrics, Toyama Medical and Pharmaceutical University, 2630 Sugitani, Toyama City 930-01, Japan).

COMMENT. Age dependent factors are important in time of withdrawal of AED and in prognosis after attempted AED withdrawal. Epileptic syndromes have an age dependent onset and course, related to CNS maturation, and varying relapse rates. Most frequent relapse rates occurred in patients undergoing drug withdrawal in preadolescence, eg benign childhood epilepsy with centrotemporal spikes, and early adulthood, eg symptomatic partial epilepsies. The characteristic course of each epileptic syndrome should be considered when attempting AED withdrawal. Background activity in the EEG is also an important factor, the persistence of slow waves and decreased alpha activity indicating an increased risk of relapse.

\section{SAFETY OF INTRAVENOUS VALPROATE}

A multicenter. open-label study of the safety of intravenous sodium valproate in 318 hospitalized patients with epilepsy is reported from the NYU Hospital for Joint Diseases; MINCEP Epilepsy Care and Minnesota Epilepsy Group, MN; University of Texas, Houston; Medical College of Virginia, Richmond; Bowman-Gray School of Medicine, Winston-Salem, NC; and 